\title{
Towards an understanding of electrogenic cotransporters: structure-function relationships
}

\author{
Forster, Ian C
}

\begin{abstract}
In this animal study we evaluated the dependence of the pulmonary diffusing capacity (D L) of nitric oxide (D L,NO) on ventilator-driven increases in alveolar volume. According to the common hypothesis as to whether D L,NO reflects the diffusive properties of the alveolar-capillary membrane, D L,NO should be improved by lung expansion. However, the influence of a simultaneous raise in intrapulmonary pressure is unknown. D L,NO was determined by applying the single-breath method to four anaesthetised paralysed rabbits (weight $3.2-3.5 \mathrm{~kg}$ ) at various alveolar volumes $(38-69 \mathrm{ml}$ ) and intrapulmonary pressures $(0.15-12.5 \mathrm{mmHg})$. The animals were ventilated with room air, using a computerised ventilatory servo-system that was also employed to perform the single-breath manoeuvres. Inflation procedures were always started from residual volume (12-13.7ml), using $0.05 \% \mathrm{NO}$ in $\mathrm{N} 2$ as the indicator gas mixture. End-tidal PNO was determined by respiratory mass spectrometry. D L,NO increased simultaneously with lung expansion and with increasing intrapulmonary pressure $(\mathrm{P}<0.001)$. We conclude that the $\mathrm{D}$ L,NO provides a measure of the pulmonary diffusive properties that is not influenced by a pressure-induced impairment of pulmonary capillary blood flow
\end{abstract}

DOI: https://doi.org/10.1007/s004240100722

Posted at the Zurich Open Repository and Archive, University of Zurich

ZORA URL: https://doi.org/10.5167/uzh-156329

Journal Article

Published Version

Originally published at:

Forster, Ian C (2001). Towards an understanding of electrogenic cotransporters: structure-function relationships. Pflügers Archiv : European Journal of Physiology, 443(2):163-165.

DOI: https://doi.org/10.1007/s004240100722 


\section{Towards an understanding of electrogenic cotransporters: structure-function relationships}

Accepted: 21 August 2001 / Published online: 6 October 2001

(C) Springer-Verlag 2001

Keywords Cotransporters · Electrophysiology · Structure-function

Elucidation of the structure-function features of membrane transport proteins is the formidable challenge facing channelologists and transportologists alike. With a few notable exceptions, such as the bacterial $\mathrm{Na}^{+} / \mathrm{H}^{+}$ exchanger (NhaA), the erythrocyte water channel (AQP-1) and the bacterial $\mathrm{K}^{+}$channel (KcsA-1), membrane transport proteins have so far resisted the purification and/or crystallization attempts that would permit the determination of their 3-D structure. Meanwhile, an armamentarium of indirect biochemical and biophysical approaches is being applied to reveal the structure-function relationships of membrane proteins. One advantage of these methods is that, in general, they can be applied under physiological conditions. Therefore, they will be valuable for interpreting the crystallographic data when they become available.

Here we focus on how the application of scanning methods can address the structure-function challenge posed by electrogenic cotransporters and highlight some selected studies from the plethora of papers that has emerged recently. Electrogenic cotransporters belong to a ubiquitously expressed diverse group of membrane proteins that utilize the free energy stored in the transmembrane electrical field and electrochemical gradient of the co-substrate ion to mediate the transmembrane transport of substrate(s) specific to that cotransport protein. Like voltage- and ligand-gated ion channels, the functional (kinetic) characteristics of most identified electrogenic cotransporters can be documented in real time under defined driving force conditions applied to a single cell, using heterologous expression systems such as Xenopus oocytes. Despite their kinetic sluggishness (transport rates 3-4 orders of magnitude slower than

I.C. Forster (国)

Physiologisches Institut der Universität Zürich-Irchel,

Winterthurerstrasse 190, 8057 Zurich, Switzerland

e-mail: IForster@access.unizh.ch

Tel.: +41-1-6355059, Fax: +41-1-6355715 channels) all the techniques used to characterize ion channels can also be applied to study electrogenic cotransporters. However, in contrast to channels, the primary amino acid sequence diversity and the absence of repetitive subunits and/or structural symmetries between cotransporter families can confound the prediction of structural motifs, or domains that subserve functions such as voltage sensing, recognition sites and the transport pathways of common cosubstrates.

\section{Functional assays and kinetic schemes}

As described below, scanning methods involve perturbing the protein (site-directed mutagenesis and eventually in situ modification of specific amino acids) and observing the subsequent changes of cotransporter kinetics. It is of course tacitly assumed that the site of perturbation coincides with the site of functional importance, but allosteric effects should not be excluded either. A thorough characterization of the wild-type protein function, from which a kinetic scheme can be derived, provides an essential framework for relating structural modifications to function (kinetic transitions, identified conformational states). Kinetic schemes for cotransporters accord with either an alternating access type model [11, 19], whereby the transport process involves a major translocation of the protein, or a channel-like multi-substrate single file model [16]. Here the substrates hop between binding sites along a single transport pathway and this does not necessitate major protein conformational changes. For both schemes, simulations can provide adequate descriptions of the measured transport kinetics (e.g. [2, 16]) and to decide between each will require additional structurefunction information (see below). The alternating access model involves the translocation of intrinsic charges (empty carrier) and two electroneutral transitions: slippage (or uncoupled leak in the absence of substrate) and cotransport. These transitions are associated with a change in the preferred orientation (peri- or cytoplasmic) of the empty, partially and fully loaded carriers respectively, as 


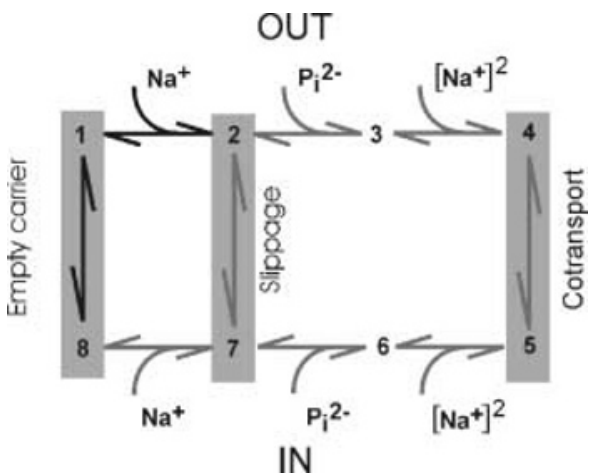

Fig. 1 A kinetic scheme for NaPi-IIa based on an alternating access model: three transitions involve reorientation of the carrier from outward- to inward-facing conformations. Transitions have been identified from steady-state and presteady-state studies (see [11]). Bold transitions are voltage dependent (adapted from [2])

illustrated for the $\mathrm{Na}^{+}$-coupled $\mathrm{P}_{\mathrm{i}}$ cotransporter $\mathrm{NaPi}$-IIa [11] in Fig. 1.

Most kinetic characterizations are based on straightforward steady state flux measurements. On the other hand, presteady-state relaxations (the analogue of ion channel gating currents), although more challenging in terms of recording technique, analysis and interpretation, can reveal subtle details associated with voltage-dependent transitions (e.g. [2, 9, 13, 17]). For many cotransporters there are no definitive data on the kinetic transitions associated with the cytosolic orientation of the protein and substrate (de-) binding order. However, techniques that allow access to the cytosol (e.g. excised patch or cut-open oocyte) and rapid light-activated concentration jumps (e.g. [17]) offer feasible approaches to rectify these two deficiencies.

\section{Scanning approaches}

By far the most widespread approach to identifying functionally important regions in cotransport proteins is the substituted cysteine accessibility method (SCAM) (e.g. [6]). The extensive application of SCAM to bacterial lac permease by the Kaback laboratory [3] has become the benchmark for application of this technique to other cotransporters in prokaryotic and eukaryotic expression systems. Methanethiosulfonate (MTS) reagents that selectively and rapidly modify cysteine residues in a hydrophilic environment can be chosen according to: (1) charge (to test for interactions with local electrical fields), (2) size and (3) membrane permeability (for defining the sidedness of the reaction). Incubation is performed under conditions (i.e. substrate and membrane potential) chosen to define the steady-state occupancy of the protein. Unlike conventional site-directed mutagenesis, in which the perturbation is predefined, given a welltolerated Cys substitution with WT-like behaviour, SCAM ideally allows one to document the effect of the Cys-modification reaction, as indicated by a change in a specific kinetic phenotype.

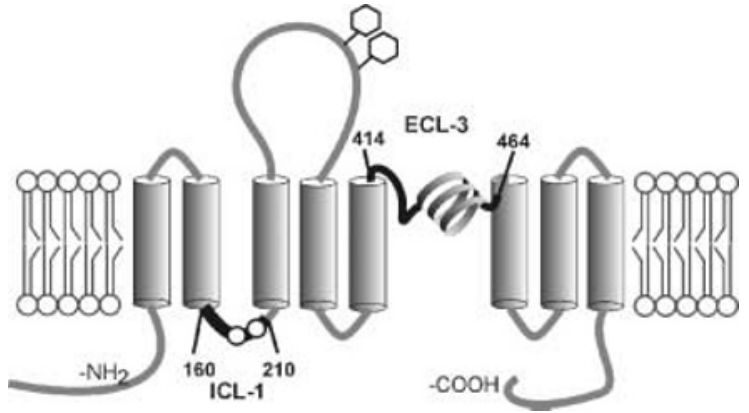

Fig. 2 The substituted cysteine accessibility method (SCAM) applied to the NaPi-IIa protein. The secondary topology was determined from hydrophobicity analysis and epitope scanning [7, 10]. External application of methanethiosulfonate (MTS) reagents identified a putative $\alpha$-helix in ECL-3 [8] and two sites in ICL-1 were accessible only by membrane-permeant MTS reagents (open circles). Bold stretches in the linkers (ICL-1, ECL-3) show high sequence homology among species [18] and may constitute the $\mathrm{P}_{\mathrm{i}}$ translocation pathway

SCAM can be used to identify functionally important sites as well as reveal and/or confirm secondary structure details, including the peri- or cytoplasmic orientation of accessible sites. For example, a functionally important region in the putative third extracellular linker (ECL-3) (see Fig. 2) of the renal $\mathrm{Na}^{+} / \mathrm{P}_{i}$ cotransporter (NaPi-IIa) [10] was probed using SCAM [8]. Incubation with impermeant MTS reagents led to the full abolition of cotransport but left the leak mode intact. The MTS-Cys reaction rates followed a periodic accessibility pattern compatible with an $\alpha$-helical motif as part of a putative reentrant loop accessible from the periplasm. A homologous region, with at least two sites accessible by MTS reagents from the cytoplasm [predicted by hydrophobicity analysis as part of the first intracellular linker (ICL-1) (see Fig. 2)], has now been identified (K. Köhler, unpublished observations). A current working hypothesis is that the NaPi-IIa cotransport pathway is formed by the association of these two linking regions from the N- and C-terminal halves of the cotransporter. In addition to ion channels (e.g. the KscA potassium channel [1] and cyclic nucleotide activated channel [12]), re-entrant loops in water-filled transport pathways appear as a recurring theme among different cotransporters, for example SGLT-1 [13] and the glutamate transporters EAAT1,2 [14]. The association of linker regions will also have important implications for determining helix-helix packing.

The characterization of even one MTS-accessible residue can also provide valuable structure-function insights into cotransport mechanisms. For example, in the Wright laboratory, charge movements were found to correlate with the accessibility of a Cys residue to MTS reagents located in the putative sugar translocation domain of the sodium glucose cotransporter SGLT-1 [9]. They elegantly combined electrophysiology with fluorescence measurements to document real-time correlations between conformational changes and charge movements. Their findings provide strong support for an alternating access scheme for $\mathrm{Na}^{+}$-driven cotransporters. 
An important caveat for unambiguous interpretation of SCAM results is that the substituted Cys residue does not lead to the exposure of a native, modifiable Cys residue (e.g. [5]), i.e. the lack of reaction of the WT protein is not necessarily sufficient grounds to proceed with SCAM and, if feasible, a Cys-free background is certainly desirable. Furthermore, although Cys substitutions are generally well tolerated, they can also lead to unexpected changes in kinetic characteristics that should be taken into account when interpreting data. These kinetic changes can provide valuable clues to identify functionally important sites. For example, for NaPi-IIa, Cys substitutions have revealed functional hot spots that confer voltage and $\mathrm{pH}$ dependence to this cotransporter [8].

Finally, we should mention two emerging scanning variants, recently applied to the shaker $\mathrm{K}^{+}$channel expressed in Xenopus oocytes, which may also prove useful for cotransporter structure-function studies. Histidine scanning involves substituting a His residue for a native charged residue and titrating it with external or internal protons to map the movements of charged residues - and voltage sensor orientation - during the channel activation process [15]. Tryptophan scanning, in which bulky Trp residues are substituted along putative membrane-spanning domains, can be used to confirm their $\alpha$-helical nature and distinguish between neighbouring helix and lipid-phase orientations of transmembrane domains, since residues facing the lipid phase show greater tolerance to these substitutions [4].

Acknowledgements The author is grateful for valuable insights from Heini Murer, Katja Köhler and Jürg Biber, technical contributions by Gerti Stange and critical comments from Marija Zečević.

\section{References}

1. Doyle DA, Cabral JM, Pfuetzer RA, Kuo A, Gulbis JM, Cohen SL, Chait BT, MacKinnon R (1998) The structure of the potassium channel: molecular basis for $\mathrm{K}^{+}$conduction and selectivity. Science 280:69-77

2. Forster IC, Biber J, Murer H (2000) Proton-sensitive transitions of the renal type II $\mathrm{Na}^{+}$coupled phosphate cotransporter kinetics. Biophys J 79:215-230
3. Frillingos S, Sahin-Toth M, Wu J, Kaback HR (1998) Cysscanning mutagenesis: a novel approach to structure-function relationships in polytopic membrane proteins. FASEB J 12: 1281-1299

4. Hong KH Miller C (2000) The lipid-protein interface of a shaker $\mathrm{K}^{+}$channel. J Gen Physiol 115:51-58

5. Kamdar G, Penado KMY, Rudnick G, Stephan MM (2001) Functional role of critical stripe residues in transmembrane span 7 of the serotonin transporter. J Biol Chem 276: 4038-4045

6. Karlin A, Akabas MH (1998) Substituted-cysteine accessibility method. Methods Enzymol 293:123-145

7. Lambert G, Traebert M, Hernando N, Biber J Murer M (1999) Studies on the topology of the renal type II NaPi-cotransporter. Pflügers Arch 437:972-978

8. Lambert G, Forster IC, Stange G, Köhler K, Biber J, Murer H (2001) Cysteine mutagenesis reveals novel structure-function features within the predicted $3^{\text {rd }}$ extracellular loop of the type IIa $\mathrm{Na}^{+} / \mathrm{P}_{\mathrm{i}}$ cotransporter. J Gen Physiol 117:533-546

9. Loo DDF, Hirayama BA, Gallardo EM, Lam JT, Turk E, Wright EM (1998) Conformational changes couple $\mathrm{Na}^{+}$and glucose transport. Proc Natl Acad Sci USA 95:7785-7794

10. Murer H, Biber J (1997) A molecular view of proximal tubular inorganic $\left(\mathrm{P}_{\mathrm{i}}\right)$ reabsorption and of its regulation. Pflügers Arch 433:379-389

11. Murer H, Hernando N, Forster I, Biber J (2000) Proximal tubular phosphate reabsorption: Molecular mechanisms. Physiol Rev 80:1373-1409

12. Roncaglia P, Becchetti A (2001) Cyclic-nucleotide-gated channels: pore topology in desensitizing E19A mutants. Pflügers Arch 441:772-780

13. Silverman M (2000) Glucose reabsorption in the kidney: molecular mechanism of the $\mathrm{Na}^{+}$/glucose cotransporter. In: Seldin DW, Giebisch G (eds) The Kidney, physiology and pathophysiology, Raven, New York, pp 2167-77

14. Slotboom DJ, Konings WN, Lolkema JS (2001) The structure of glutamate transporters shows channel-like features. FEBS Lett 492:183-186

15. Storace DM, Bezanilla F (2001) Histidine scanning mutagenesis of basic residues of the $\mathrm{S} 4$ segment of the shaker $\mathrm{K}^{+}$channel. J Gen Physiol 117:469-490

16. Su A, Mager S, Mayo SL, Lester HA (1996) A multi-substrate single-file model for ion-coupled transporters. Biophys J 70: 762-777

17. Watzke N, Bamberg E, Grewer C (2001) Early intermediates in the transport cycle of the neuronal excitatory amino acid carrier EAAC1. J Gen Physiol 117:547-562

18. Werner A, Kinne RKH (2001) Evolution of the Na- $\mathrm{P}_{i}$ cotransport systems. Am J Physiol 280:R301-R312

19. Wright EM (2001) Renal $\mathrm{Na}^{+}$glucose transporters. Am J Physiol 280:F10-F18 